23

\title{
Зондирование молекул сероводорода лидаром комбинационного рассеяния света с летающей платформы
}

\author{
(C) В.Е. Привалов ${ }^{1}$, В.Г. Шеманин ${ }^{2}$ \\ ${ }^{1}$ Санкт-Петербургский политехнический университет Петра Великого, \\ 195251 Санкт-Петербург, Россия \\ ${ }^{2}$ Новороссийский политехнический институт, фрилиал Кубанского государственного технологического университета, \\ 353900 Новороссийск, Россия \\ e-mail: vaevpriv@yandex.ru
}

Поступила в редакцию 14.05.2018 г.

В окончательной редакции 02.07.2018 г.

\begin{abstract}
Численно решено лидарное уравнение для комбинационного рассеяния света молекулами сероводорода в направлении $180^{\circ}$ при зондировании молекул сероводорода в атмосфере и воде с концентрациями в диапазоне $10^{11}-10^{15} \mathrm{~cm}^{-3}$ с летающей платформы на высотах до $500 \mathrm{~m}$ в режиме синхронного счета фотонов. Показано, что время измерения для такого уровня концентраций исследуемых молекул на глубине до $100 \mathrm{~m}$, на длине волны лазерного излучения $532 \mathrm{~nm}$ и высоте платформы до $500 \mathrm{~m}$ не превышает $34 \mathrm{~min}$. Разработка новых схем лидаров открывает дополнительные возможности для дистанционного зондирования низких концентраций молекул сероводорода в морской воде.
\end{abstract}

DOI: $10.21883 / O S .2018 .10 .46714 .129-18$

Как известно [1], в Черном море существует обширный слой жидкого сероводорода, который расположен в разных частях моря на глубинах от 90 до $150 \mathrm{~m}$. Уровень сероводородного слоя постоянно пульсирует, поднимается иногда до уровня $75 \mathrm{~m}$, потом снижается. Поэтому возможно повышение концентрации молекул сероводорода в морской воде и загрязнение ими атмосферного воздуха над морем. Все это представляет серьезную проблему в связи с высокой токсичностью и взрывоопасностью сероводорода.

Мониторинг газовых молекул с летающей платформы может стать эффективным способом решения этой проблемы за счет большой площади охвата на поверхности моря или большого измерительного объема в атмосферном пограничном слое и возможности получить больше информации за короткий промежуток времени [2]. Поэтому становится актуальной задача разработки новых лидаров для измерения концентрации молекул $\mathrm{H}_{2} \mathrm{~S}$ на уровне от предельно допустимых концентраций (ПДК) $0.008 \mathrm{mg} / \mathrm{m}^{3}$ [3] или $1.4 \cdot 10^{11} \mathrm{~cm}^{-3}$ до тысяч ПДК, причем не только в атмосферном воздухе, но и в воде.

Ранее [2-4] были рассмотрены методы лазерного зондирования молекул сероводорода в атмосфере. Было показано, что лидар комбинационного рассеяния света (КРС) может использоваться для дистанционного измерения концентрации молекул на уровне сотен и тысяч ПДК. Представляет интерес теоретическая оценка возможностей лидара КРС для обнаружения молекул сероводорода в воде на уровне концентрации порядка ПДК и выше с летающей платформы в диапазоне высот до $500 \mathrm{~m}$. Причем в качестве летающей платформы может рассматриваться любой летательный аппарат, включая беспилотные. Поэтому целью настоящей работы является оценка параметров лидара КРС для зондирования молекул сероводорода в воде и в атмосферном пограничном слое на уровне ПДК и выше с летающей платформы на высотах порядка сотен метров в режиме синхронного счета фотонов.

Для этого было выполнено численное решение лидарного уравнения для КРС молекулами сероводорода в направлении $180^{\circ}$ для выбора длины волны лазерного излучения, параметров лидара и получения минимального времени измерения сигнала комбинационного рассеяния света.

Оптическая схема такого лидара подробно рассмотрена в $[2,3]$ и приведена на рис. 1.

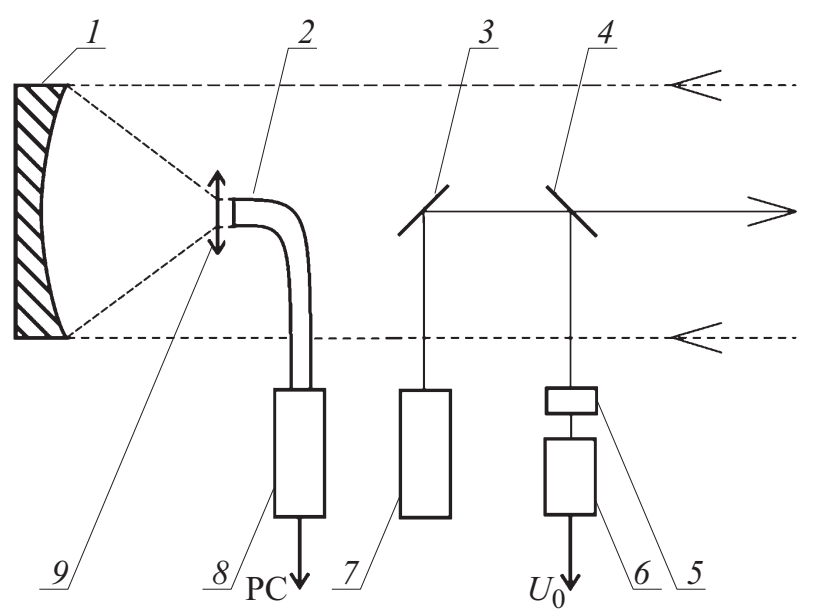

Рис. 1. Оптическая схема лидара КРС: 1 - сферическое зеркало приемного телескопа, 2 - волоконный ввод, 3 глухое зеркало, 4 - стеклянная пластина, 5 - интерференционный светофильтр, 6 - фотоприемник, 7 - лазер, 8 микроспектрометр, 9 - линзовый объектив. 
В качестве возможных вариантов рассмотрим использование второй гармоники YAG: Nd-лазера (длина волны $532 \mathrm{~nm}$ ) с накачкой полупроводниковым лазером и полупроводниковых лазеров с длинами волн 405 и $655 \mathrm{~nm} \mathrm{c}$ длительностью импульсов $10 \mathrm{~ns}$ и энергиями в импульсе до $1 \mathrm{~mJ}$ при частоте следования лазерных импульсов $f$ до $1 \mathrm{MHz}$ [2]. Излучение лазера 7 направлялось вдоль оси приемного телескопа перпендикулярно поверхности моря. Излучение комбинационного рассеяния молекулами сероводорода в атмосферном пограничном слое и в воде в направлении назад собиралось приемным телескопом типа Ньютона со сферическим зеркалом 1 диаметром $400 \mathrm{~mm}$ и фокусировалось линзовым объективом 9 в волоконный ввод 2 микроспектрометра 8 типа FSD-8, работающего на линии с ПК. Часть лазерного излучения направлялась стеклянной пластиной 4 через интерференционный светофильтр 5 на фотодиод 6 , напряжение $U_{0}$ с которого записывалось платой сбора данных в ПК для контроля энергии лазерного импульса и формирования начала отсчета времени измерения.

Лидарное уравнение для КРС исследуемыми молекулами в направлении назад в режиме счета фотонов запишем для чисел фотонов в виде [2]

$$
\begin{aligned}
n(\lambda, H)= & n_{0} K_{1} \Delta H G(H) f t S_{0} T_{0}\left(\lambda_{0}, H\right) \\
& \times T(\lambda, H) N(H)(d \sigma / d \Omega) / 2 H^{2}
\end{aligned}
$$

где $n(\lambda, H)$ - число фотонов, зарегистрированное фотодетектором лидара на длине волны КРС $\lambda$ с высоты $H$, $n_{0}$ - число фотонов на длине волны лазерного излучения $\lambda_{0}, K_{1}$ - лидарная константа на длине волны $\lambda$, $S_{0}$ - площадь приемной апертуры телескопа, $G(H)-$ геометрическая функция лидара, $N(H)$ - концентрация исследуемых молекул, $t$ - время измерения, сомножители (пропускание атмосферы) равны соответственно [5]

$$
\begin{gathered}
T_{0}\left(\lambda_{L}, H\right)=\exp \left[-\int_{0}^{H} \alpha\left(\lambda_{L}, z\right) d z\right], \\
T(\lambda, H)=\exp \left[-\int_{0}^{H} \alpha(\lambda, z) d z\right],
\end{gathered}
$$

где $\alpha\left(\lambda_{L}, z\right)$ и $\alpha(\lambda, z)$ - коэффициенты ослабления на частотах лазерного излучения и КРС молекулами сероводорода, $d \sigma / d \Omega$ - дифференциальное сечение КРС-исследуемыми молекулами.

Будем рассматривать однократное комбинационное рассеяние и однородную среду (атмосферу или морскую воду) $[3,5]$. Тогда из уравнения (1) выразим время измерения, которое будет служить мерой эффективности лидара, в виде

$$
\begin{aligned}
t= & n(\lambda, H) H^{2} /\left[n_{0} K_{1} \Delta H G(H) f S_{0} T_{0}\left(\lambda_{0}, H\right)\right. \\
& \times T(\lambda, H) N(H)(d \sigma / d \Omega)] .
\end{aligned}
$$

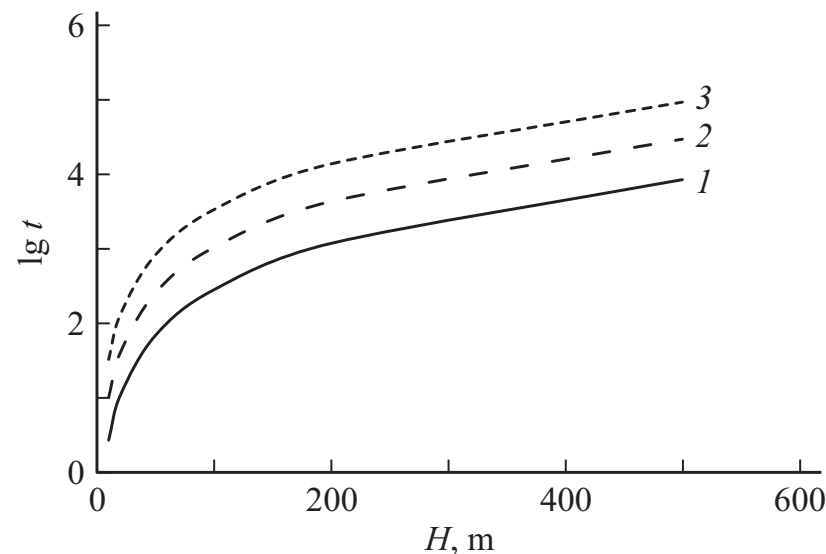

Рис. 2. Логарифмическая зависимость времени измерения $t$ (в s) от расстояния $H$ (в $\mathrm{m})$, рассчитанная по уравнению (2) для длин волн лазерного излучения 405 (1), 532 (2) и $655 \mathrm{~nm}$ (3) при зондировании молекул сероводорода в атмоcфepe.

Для нашей экспериментальной ситуации возьмем концентрацию молекул сероводорода в диапазоне $N(z)=10^{11}-10^{15} \mathrm{~cm}^{-3}$. Значение максимума полосы валентных колебаний $\mathrm{SH}$ молекул $\mathrm{H}_{2} \mathrm{~S}$ взято из [3] и равно $2611 \mathrm{~cm}^{-1}$. Значения коэффициентов ослабления в атмосфере и воде для этих длин волн $\alpha\left(\lambda_{L}, z\right)$ и $\alpha(\lambda, z)$, дифференциальных сечений колебательного КРС собраны из $[3,6]$ в таблице.

Поперечное сечение зеркала приемного телескопа лидара было $0.13 \mathrm{~m}^{2}$, а шаг по расстоянию $-7.5 \mathrm{~m}$. Лидарная константа $K_{1}$ определялась как произведение спектрального пропускания приемного телескопа на относительную спектральную чувствительность фотоприемника $\xi(\lambda)$ на длине волны $\lambda[3]$ из таблицы.

Рассмотрим численное решение уравнения (2) в предположении, что $G(H)$ для нашего случая равно 1 , концентрация молекул сероводорода $N(z)=10^{15} \mathrm{~cm}^{-3}$ и за время измерения $t$ будут зарегистрированы 10 фотонов. Результаты решения уравнения (2) для этого случая представлены на рис. 2.

Как следует из графика рис. 2, спектральные зависимости величин, входящих в уравнение (2), приводят к уменьшению времени измерения в ряду длин волн лазерного излучения $655-532-405 \mathrm{~nm}$, но за первые $100 \mathrm{~m}$ это время измерения резко нарастает.

Минимальное время измерения можно получить на длине волны $405 \mathrm{~nm}$ : для $50 \mathrm{~m}$ это значение составит почти $70 \mathrm{~s}$, для $100 \mathrm{~m}-285 \mathrm{~s}$, а для $500 \mathrm{~m}-$ почти $850 \mathrm{~s}$ при частоте следования лазерных импульсов $1 \mathrm{MHz}$ и времени одного измерения $50 \mathrm{~ns}$ (шаг по высоте $\Delta H=7.5 \mathrm{~m})$. Очевидно, что уменьшение времени измерения возможно за счет увеличения частоты следования лазерных импульсов или увеличения их энергии.

Рассмотрим зависимость времени измерения от концентрации исследуемых молекул. На рис. 3 приведено решение уравнения (2) для концентрации молекул серо- 
Длины волн лазерного излучения и полос КРС-молекул сероводорода, дифференциальные сечения колебательного КРС для этих полос, коэффициенты ослабления в атмосфере $(a)$ и в воде $(w)$ на этих длинах волн и относительная спектральная чувствительность фотоприемника

\begin{tabular}{l|c|c|c|c|c|c}
\hline \multirow{2}{*}{$\begin{array}{c}\text { Длины } \\
\text { волн, } \mathrm{nm}\end{array}$} & $\begin{array}{c}\text { Дифференциальное } \\
\begin{array}{c}\text { сечение КР } \\
(d \sigma / d \Omega) \cdot 10^{30} \mathrm{~cm}^{2}\end{array}\end{array}$ & $\begin{array}{c}\alpha_{a}\left(\lambda_{0}, z\right), \\
\mathrm{km}^{-1}\end{array}$ & $\begin{array}{c}\alpha_{a}(\lambda, z), \\
\mathrm{km}^{-1}\end{array}$ & $\begin{array}{c}\alpha_{w}\left(\lambda_{0}, z\right), \\
\mathrm{km}^{-1}\end{array}$ & $\begin{array}{c}\alpha_{w}(\lambda, z), \\
\mathrm{km}^{-1}\end{array}$ & $\begin{array}{c}\text { Относительная спектральная } \\
\text { чувствительность ФП } \xi(\lambda)\end{array}$ \\
\cline { 3 - 6 } $\begin{array}{l}655 \\
790.1\end{array}$ & 1.35 & 0.14 & 0.08 & 0.15 & 0.28 \\
532 & 3.10 & 0.16 & 0.11 & 0.02 & 0.07 & 0.48 \\
617.8 & 9.23 & 0.24 & 0.15 & 0.05 & 0.04 & 0.8 \\
405 & & & 0.21 & &
\end{tabular}

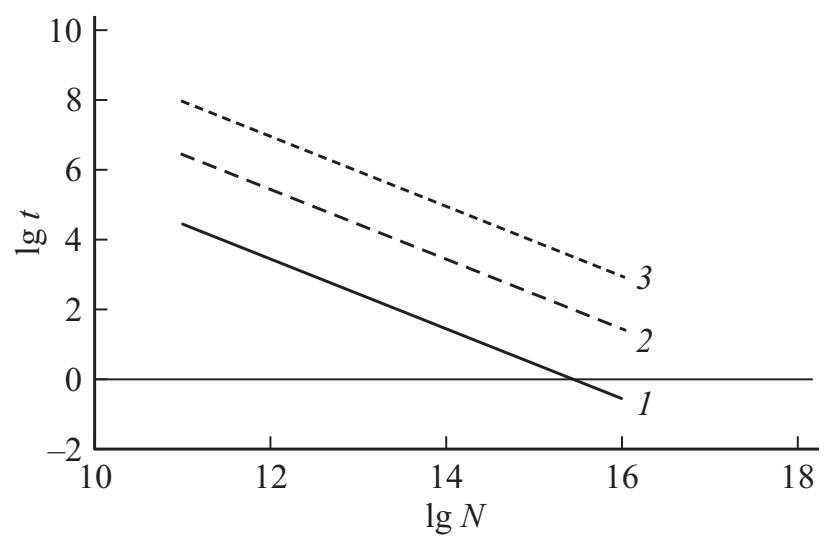

Рис. 3. Логарифмическая зависимость времени измерения $t$ (в $\mu \mathrm{s})$ от $\lg N\left(N\right.$ в $\left.\mathrm{cm}^{-3}\right)$, рассчитанная по уравнению (2) для длины волны лазерного излучения $405 \mathrm{~nm}$ при зондировании молекул сероводорода в атмосфере с высоты 10 (1), 100 (2) и $500 \mathrm{~m}(3)$.

водорода от $10^{11}$ до $10^{16} \mathrm{~cm}^{-3}$ и высот 10,100 и $500 \mathrm{~m}$, на которых летают различные летательные аппараты. В этом случае время, необходимое для регистрации сигнала с концентрацией молекул $10^{11} \mathrm{~cm}^{-3}$ и высоты $100 \mathrm{~m}$, составляет для длины волны $405 \mathrm{~nm} 2.9$ s, а высоты $500 \mathrm{~m}-85 \mathrm{~s}$. При этом за $85 \mathrm{~s}$ смещение летательного аппарата, движущегося со скоростью $10 \mathrm{~m} / \mathrm{s}$, составит $850 \mathrm{~m}$ и приведет к увеличению измерительного объема до почти $595000 \mathrm{~m}^{3}$ с высотой $500 \mathrm{~m}$, при этом на $7.5 \mathrm{~m}$ высоты придется около $9000 \mathrm{~m}^{3}$. Движение летающей платформы приводит к заметному увеличению измерительного объема с 769 до $595000 \mathrm{~m}^{3}$ или почти в 800 раз по сравнению с неподвижной платформой.

Из уравнения (2) выразим время измерения для зондирования под водой в виде

$$
\begin{aligned}
t= & 4 n\left(\lambda, H_{a}, H_{w}\right) \pi n_{w}^{2}\left(H_{a}+H_{w} / n_{w}\right)^{2} /\left[n_{0} K_{1} \Delta H G(H)\right. \\
& \times k_{w} f S_{0} N\left(H_{w}\right)(d \sigma / d \Omega) T_{0}\left(\lambda_{0}, H_{a}\right) T\left(\lambda, H_{a}\right) \\
& \left.\times T_{0 w}\left(\lambda_{0}, H_{w}\right) T_{w}\left(\lambda, H_{w}\right)\right] .
\end{aligned}
$$

Результаты расчетов по уравнению (3) для зондирования молекул сероводорода с концентрацией $10^{15} \mathrm{~cm}^{-3}$ на длине волны лазерного излучения $405 \mathrm{~nm}$ и глубин до 100 m приведены на рис. 4. Эти графики показывают, что увеличение глубины зондирования моря на $10 \mathrm{~m}$ при заданной концентрации исследуемых молекул ведет к увеличению времени измерения от $1.8 \mathrm{~s}$ для высоты платформы $100 \mathrm{~m}$ до $4.7 \mathrm{~s}$ для $500 \mathrm{~m}$. Результаты расчетов для трех длин волн лазерного излучения в зависимости от глубины зондирования в воде представлены на рис. 5. Полученное различие связано с различием в двухпроходных коэффициентах пропускания границы раздела воздух-вода по данным [6]. Для расчетов были взяты из [6] значения для длины волны $405 \mathrm{~nm}-$ $0.2 \mathrm{~m}^{-1}, 532 \mathrm{~nm}-0.1 \mathrm{~m}^{-1}$, а для $655 \mathrm{~nm}-0.35 \mathrm{~m}^{-1}$, что и привело к невозможности зондирования на длине волны лазерного излучения $655 \mathrm{~nm}$ на глубинах более $60 \mathrm{~m}$.

Выполним расчеты зависимости времени измерения от концентрации исследуемых молекул для пяти значений глубины зондирования в воде на длине волны

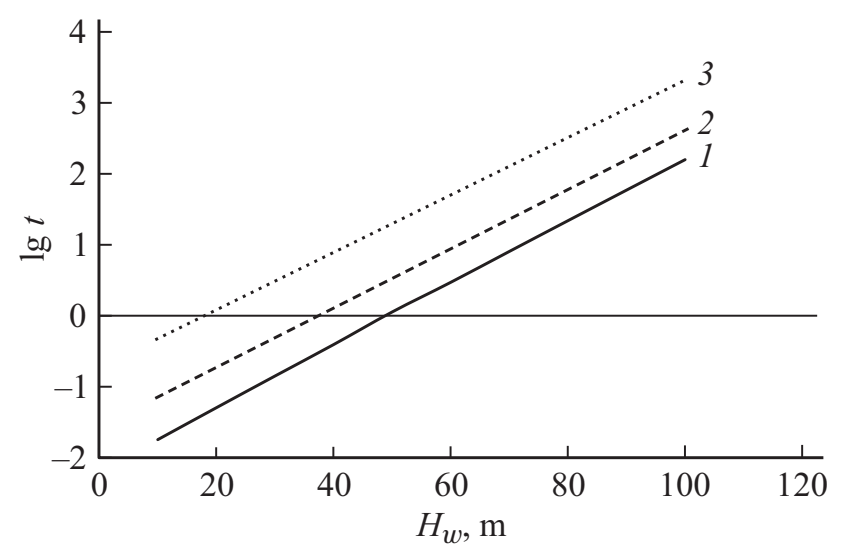

Рис. 4. Логарифмическая зависимость времени измерения $t$ (в s) от глубины $H_{w}$ (в m), рассчитанная по уравнению (3) для длины волны лазерного излучения $405 \mathrm{~nm}$ при зондировании молекул сероводорода с концентрацией $10^{15} \mathrm{~cm}^{-3}$ с движущейся летающей платформы на высотах 10 (1), 100 (2) и 500 m (3) над уровнем моря. 


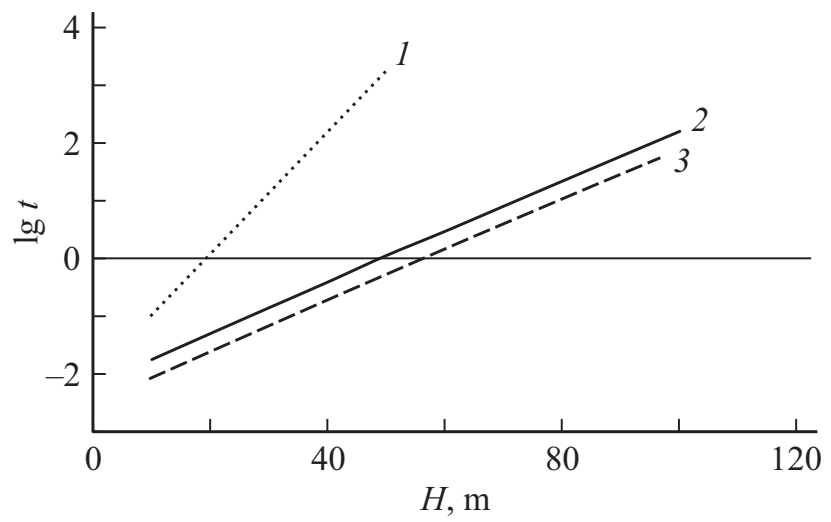

Рис. 5. Логарифмическая зависимость времени измерения $t$ (в s) от глубины $H_{w}$ (в $\mathrm{m}$ ), рассчитанная по уравнению (3) для трех длин волн лазерного излучения 405 (2), 532 (3) и $655 \mathrm{~nm}$ (1) при зондировании молекул сероводорода с концентрацией $10^{15} \mathrm{~cm}^{-3}$ с движущейся летающей платформы на высоте $100 \mathrm{~m}$ над уровнем моря.

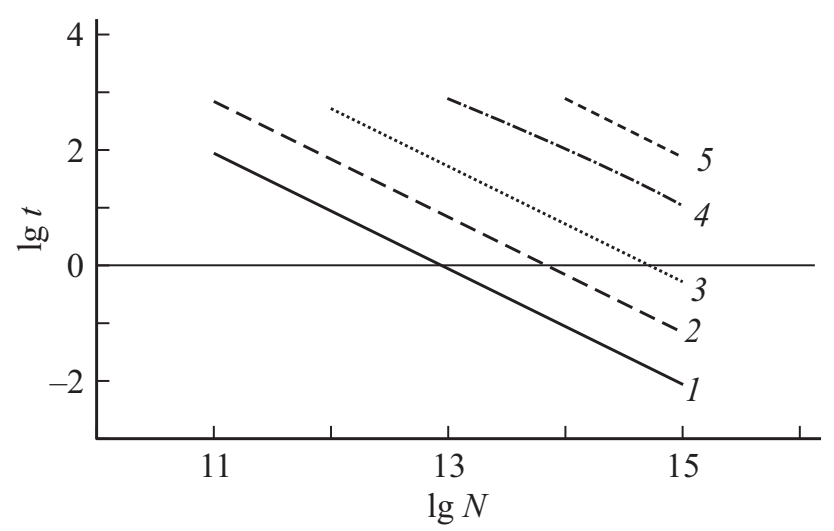

Рис. 6. Логарифмическая зависимость времени измерения $t$ (в s) от $\lg N\left(N\right.$ в $\left.\mathrm{cm}^{-3}\right)$, рассчитанная по уравнению (3) для глубин $H_{w}=10(1), 30(2), 50$ (3), 80 (4), 100 m (5) и длины волны лазерного излучения $532 \mathrm{~nm}$ при зондировании с летающей платформы на высоте $100 \mathrm{~m}$ над уровнем моря.

лазерного излучения $532 \mathrm{~nm}$, причем время измерения было ограничено сверху значением 1000 s. Эта логарифмическая зависимость времени измерения от логарифма концентрации молекул сероводорода представлена на рис. 6. Представленный на рис. 6 результат свидетельствует о невозможности зондирования молекул сероводорода с концентрациями менее $10^{13} \mathrm{~cm}^{-3}$ на длине волны лазерного излучения $532 \mathrm{~nm}$ на глубинах более $80 \mathrm{~m}$.

Таким образом, анализ полученных результатов показывает, что время измерения для уровня концентрации исследуемых молекул сероводорода $10^{15} \mathrm{~cm}^{-3}$ на длине волны лазерного излучения $532 \mathrm{~nm}$, высоте полета платформы до $500 \mathrm{~m}$ и глубине зондирования в толщу воды до $100 \mathrm{~m}$ достигает $2030 \mathrm{~s}$ для принятого варианта лидара КРС. Поэтому можно вести речь о регистрации таким лидаром с летающей платформы за разумные времена концентрации исследуемых молекул сероводорода на уровне ПДК с высоты $100 \mathrm{~m}$. Следовательно, можно будет зондировать молекулы сероводорода в воде с концентрациями на уровне ПДК и выше с высоты до $100 \mathrm{~m}$.

Полученные результаты хорошо согласуются с данными [6] и отражают различие в уровнях ПДК исследованных молекул углеводородов [3], а разработка новых вариантов лидаров, например [2], открывает потенциальные возможности для дистанционного зондирования низких концентраций молекул сероводорода в воде с летающей платформы.

Работа была частично поддержана в рамках Основной части Гос. задания Министерства образования и науки РФ № 5.7721.2017/БЧ.

\section{Список литературы}

[1] Иванов А. Из Черного моря уходит жизнь.

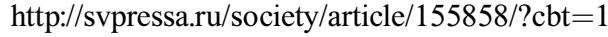

[2] Привалов В.Е., Шеманин В.Г. // Опт. и спектр. 2017. T. 123. № 6. C. 941.

[3] Привалов В.Е., Фотиади А.Э., Шеманин В.Г. Лазеры и экологический мониторинг атмосферы. СПб.: Лань, 2013. $288 \mathrm{c}$.

[4] Privalov V.E., Shemanin V.G. // Proc. SPIE. 1998. V. 3345. P. 6.

[5] Донченко В.А., Кабанов М.В., Кауль Б.В., Самохвалов И.В. Атмосферная электрооптика. Томск: Изд-во НТЛ, 2010. C. $178-181$.

[6] Межкерис Р. Лазерное дистанционное зондирование. М.: Мир, 1987. $550 \mathrm{c}$. 